\title{
State tomography for two qubits using reduced densities
}

\author{
D. Petz ${ }^{1,3}$, K.M. Hangos ${ }^{2,3}$, A. Szántó ${ }^{1,3}$ and F. Szöllősi ${ }^{1,3}$ \\ ${ }^{1}$ Budapest University of Technology and Economics \\ ${ }^{2}$ Computer and Automation Research Institute
}

\begin{abstract}
The optimal state determination (or tomography) is studied for a composite system of two qubits when measurements can be performed on one of the qubits and interactions of the two qubits can be implemented. The goal is to minimize the number of interactions to be implemented. The algebraic method used in the paper leads to an extension of the concept of mutually unbiased measurements.
\end{abstract}

PACS numbers: 03.67.-a, 03.65.Wj, 03.65.Fd

Key words: State determination, reduced density, unbiased measurement, minimal realization, unbiased subalgebra, Pauli matrices.

\section{Introduction}

An $n$-level quantum system is described by an $n$-dimensional Hilbert space $\mathcal{H}$, or equivalently by the algebra $M_{n}(\mathbb{C})$ of the $n \times n$ complex matrices. When an orthogonal basis of $\mathcal{H}$ is chosen, operators acting on $\mathcal{H}$ correspond to $n \times n$ matrices. A positive operator $\rho$ of trace 1 is called state. If we choose and fix an orthonormal basis $\left\{e_{1}, e_{2}, \ldots, e_{n}\right\}$, then a state $\rho$ is determined by the matrix elements $\rho_{i j}=\left\langle e_{i}|\rho| e_{j}\right\rangle$. Determination of $\rho$ involves $n^{2}-1$ real parameters, namely, $\rho_{i i}(1 \leq i \leq n-1)$, $\operatorname{Re} \rho_{i j}$ and $\operatorname{Im} \rho_{i j}(1 \leq i<j \leq n)$.

A von Neumann measurement on the system is a family $\mathcal{M}=\left\{P_{1}, P_{2}, \ldots, P_{d}\right\}$ of pairwise orthogonal projections such that $\sum_{i} P_{i}=I$. When the measurement $\mathcal{M}$ is performed in the state $\rho$, the outcome $1 \leq j \leq d$ appear with probability $p_{j}=\operatorname{Tr} \rho P_{i}$ for each $j[5,6]$. Independent measurements on several copies of our quantum system give the relative frequencies $f_{j}$ for each outcome $j$ and $f_{j}$ is an estimate of the probability $p_{j}$.

\footnotetext{
${ }^{3}$ Supported by the Hungarian Research Grants OTKA T042710, T063066 and T032662.
} 
The repeated measurement provides $d-1$ degree of freedom concerning the the density $\rho$, since $\sum_{j} p_{j}=1$. The information we obtained is maximal if $d=n$ which means that all the projections $P_{j}$ are of rank one. $\rho$ is determined by $n^{2}-1$ parameters, hence at least $n+1$ different measurements are to be performed to cover all degrees of freedom. Of course, the $n+1$ different measurements are sufficient in the case when they provide "non-overlapping" information.

The literature of state tomography is very rich, there are several protocols, and the efficiency of state reconstruction can be increased if the later measurements depend on the outcomes of the former ones [1, 7, 9].

The composite system of two qubits is a 4-level quantum system which is described on the space $\mathbb{C}^{4} \equiv \mathbb{C}^{2} \otimes \mathbb{C}^{2}$. A state is described by 15 real parameters. Therefore, at least 5 kinds of elementary measurements should be made to determine the state of the system.

Denote by $A$ and $B$ the two qubits. Then $M_{4}(\mathbb{C})=B\left(\mathcal{H}_{A}\right) \otimes B\left(\mathcal{H}_{B}\right)$, where $B\left(\mathcal{H}_{A}\right)$ and $B\left(\mathcal{H}_{B}\right)$ are isomorphic to $M_{2}(\mathbb{C})$. Assume that we can perform measurements only on the qubit $A$. If the total system has the statistical operator $\rho_{A B}$, then we can reconstruct the reduced density

$$
\rho_{A}^{(1)}:=\operatorname{Tr}_{B} \rho_{A B}
$$

after some measurements. In order to get more information, we switch on an interaction between the two qubits. If $H$ is the Hamiltonian, then the new state is

$$
e^{\mathrm{i} H} \rho_{A B} e^{-\mathrm{i} H}=W_{1} \rho_{A B} W_{1}^{*}
$$

after the interaction. (For the sake of simplicity, the interaction is kept for a time unit.) The new reduced density is

$$
\rho_{A}^{(2)}:=\operatorname{Tr}_{B} W_{1} \rho_{A B} W_{1}^{*} .
$$

This procedure may be continued by using other interactions and ends with a sequence of reduced states $\rho_{A}^{(1)}, \rho_{A}^{(2)}, \ldots, \rho_{A}^{(k)}$. We want to determine the minimal $k$ such that this sequence of reduced densities determines $\rho_{A B}$. In other words, we want to minimize the number of interactions between the two qubits. It turns out that the minimum number is 5 .

Minimal realizations play an important role in systems theory, too [3], because they represent the state of the system with the minimum possible number of parameters. Minimal realizations are known to be jointly controllable and observable for most of the known system classes. The above problem of finding the minimum number of reduced states can be regarded as a minimal representation problem for a system that consists of a pair of coupled qubits.

\section{Algebraic formulation}

Instead of the transformation (11) of the density matrix $\rho_{A B}$, we can change the subalgebra and we have an equivalent algebraic formulation. The total system is described by the 
algebra $M_{4}(\mathbb{C})$. We look for subalgebra $\mathcal{A}_{1}, \mathcal{A}_{2}, \ldots, \mathcal{A}_{k}$ such that

1. Each $\mathcal{A}_{j}$ is algebraically isomorphic to $M_{2}(\mathbb{C}), 1 \leq j \leq k$.

2. The linear span of the subspaces $\mathcal{A}_{1}, \mathcal{A}_{2}, \ldots, \mathcal{A}_{k}$ is $M_{4}(\mathbb{C})$.

Given a subalgebra $\mathcal{A}_{j}$, there is a unitary $W_{j}$ such that $W_{j}^{*} \mathcal{A}_{j} W_{j}=B\left(\mathcal{H}_{A}\right) \otimes \mathbb{C} I_{B}$. The reduced density $\rho_{j} \in \mathcal{A}_{j}$ is the same as the reduction of $W_{j} \rho_{A B} W_{j}^{*}$ to the first spin. Therefore, instead of the reduction of density after the interaction, we can work with the reduced density of $\rho_{A B}$ in $\mathcal{A}_{j}$. The second condition makes sure that the reduced densities in $\mathcal{A}_{1}, \mathcal{A}_{2}, \ldots, \mathcal{A}_{k}$ determine $\rho_{A B}$ completely.

The traceless subspace of $M_{4}(\mathbb{C})$ has dimension 15, while the traceless subspace of $\mathcal{A}_{j}$ has dimension 3 , therefore we need $k \geq 5$ to fulfill the requirements. It will turn out that $k=5$ is possible.

The algebra $M_{2}(\mathbb{C})$ is linearly spanned by the Pauli matrices:

$$
\sigma_{0}:=\left[\begin{array}{ll}
1 & 0 \\
0 & 1
\end{array}\right], \quad \sigma_{1}:=\left[\begin{array}{ll}
0 & 1 \\
1 & 0
\end{array}\right], \quad \sigma_{2}:=\left[\begin{array}{cc}
0 & -\mathrm{i} \\
\mathrm{i} & 0
\end{array}\right], \quad \sigma_{3}:=\left[\begin{array}{cc}
1 & 0 \\
0 & -1
\end{array}\right] .
$$

Recall that they satisfy the multiplication rules

$$
\sigma_{i} \sigma_{j}=\delta_{i j} I+\mathrm{i} \sum_{k=1}^{3} \epsilon_{i j k} \sigma_{k} \quad(1 \leq i, j \leq 3),
$$

where $\epsilon_{i j k}$ is the Levi-Civita tensor:

$$
\epsilon_{i_{1} i_{2} \cdots i_{n}}= \begin{cases}0 & \text { if } \exists j, k \text { such that } i_{j}=i_{k}, \\ 1 & \text { if the permutation }\left(i_{1} i_{2} \cdots i_{n}\right) \text { is even } \\ -1 & \text { if the permutation }\left(i_{1} i_{2} \cdots i_{n}\right) \text { is odd }\end{cases}
$$

The above rules can be essentially simplified by posing the following two requirements:

1. $\sigma_{j}$ is a self-adjoint unitary $(1 \leq j \leq k)$ and $\sigma_{3}=-\mathrm{i} \sigma_{1} \sigma_{2}$.

2. $\sigma_{1} \sigma_{2}+\sigma_{2} \sigma_{1}=0$.

When a triplet $\left(S_{1}, S_{2}, S_{3}\right)$ satisfies these condition, it will be called a Pauli triplet. For such a triplet $\operatorname{Tr} S_{i}=0$ and $\operatorname{Tr} S_{i} S_{j}=0$ for $i \neq j$. The latter relation is interpreted as the orthogonality of $S_{i}$ and $S_{j}$ with respect to the Hilbert-Schmidt inner product $\langle A, B\rangle:=\operatorname{Tr} A^{*} B$. Furthermore, it can be seen that the two relations above imply (2).

Given a Pauli triplet $\left(S_{1}, S_{2}, S_{3}\right)$, the linear mapping defined as

$$
\sigma_{0} \mapsto I, \quad \sigma_{1} \mapsto S_{1}, \quad \sigma_{2} \mapsto S_{2}, \quad \sigma_{3} \mapsto-\mathrm{i} S_{1} S_{2}
$$

is an algebraic isomorphism between $M_{2}(\mathbb{C})$ and the linear span of the operators $I, S_{1}, S_{2}$ and $S_{3}$.

In the algebra $M_{4}(\mathbb{C})$, the elementary tensors $\sigma_{i} \otimes \sigma_{j}$ form an orthogonal basis $(0 \leq$ $i, j \leq 3)$. All these operators are self-adjoint unitaries and can be chosen to be $S_{i}$ 's.

The next proposition is the main result of the paper. 
Proposition 1 There are 5 sublalgebras of $B\left(\mathcal{H}_{A}\right) \otimes B\left(\mathcal{H}_{B}\right)$ such that each of them is isomorphic to $M_{2}(\mathbb{C})$ and the reduced states determine an arbitrary state $\rho_{A B}$ of the two qubits $A$ and $B$.

Proof. First we take the following Pauli triplets consisting of elementary tensors:

$$
\begin{aligned}
& \left\{\sigma_{0} \otimes \sigma_{1},-\sigma_{1} \otimes \sigma_{3}, \sigma_{1} \otimes \sigma_{2}\right\}
\end{aligned}
$$

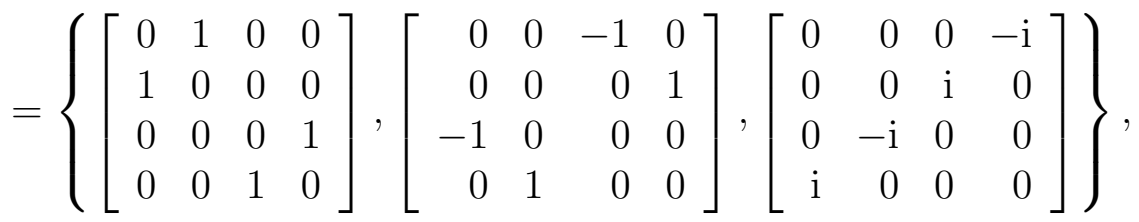

$$
\begin{aligned}
& \left\{\sigma_{3} \otimes \sigma_{1}, \sigma_{1} \otimes \sigma_{1}, \sigma_{2} \otimes \sigma_{0}\right\} \\
& =\left\{\left[\begin{array}{rrrr}
0 & 1 & 0 & 0 \\
1 & 0 & 0 & 0 \\
0 & 0 & 0 & -1 \\
0 & 0 & -1 & 0
\end{array}\right],\left[\begin{array}{llll}
0 & 0 & 0 & 1 \\
0 & 0 & 1 & 0 \\
0 & 1 & 0 & 0 \\
1 & 0 & 0 & 0
\end{array}\right],\left[\begin{array}{rrrr}
0 & 0 & -\mathrm{i} & 0 \\
0 & 0 & 0 & -\mathrm{i} \\
\mathrm{i} & 0 & 0 & 0 \\
0 & \mathrm{i} & 0 & 0
\end{array}\right]\right\}, \\
& \left\{\sigma_{1} \otimes \sigma_{0}, \sigma_{2} \otimes \sigma_{2}, \sigma_{3} \otimes \sigma_{2}\right\}
\end{aligned}
$$

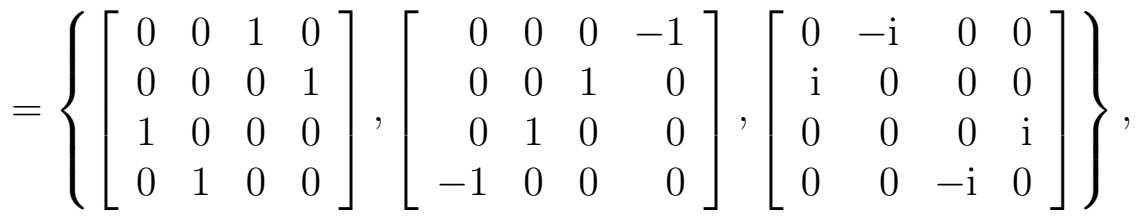

$$
\begin{aligned}
& \left\{\sigma_{0} \otimes \sigma_{2}, \sigma_{2} \otimes \sigma_{3}, \sigma_{2} \otimes \sigma_{1}\right\} \\
& =\left\{\left[\begin{array}{rrrr}
0 & -\mathrm{i} & 0 & 0 \\
\mathrm{i} & 0 & 0 & 0 \\
0 & 0 & 0 & -\mathrm{i} \\
0 & 0 & \mathrm{i} & 0
\end{array}\right],\left[\begin{array}{rrrr}
0 & 0 & -\mathrm{i} & 0 \\
0 & 0 & 0 & \mathrm{i} \\
\mathrm{i} & 0 & 0 & 0 \\
0 & -\mathrm{i} & 0 & 0
\end{array}\right],\left[\begin{array}{rrrr}
0 & 0 & 0 & -\mathrm{i} \\
0 & 0 & -\mathrm{i} & 0 \\
0 & \mathrm{i} & 0 & 0 \\
\mathrm{i} & 0 & 0 & 0
\end{array}\right]\right\} .
\end{aligned}
$$

Together with the identity, each triplet linearly spans a subalgebra $\mathcal{A}_{j}(1 \leq j \leq 4)$. It is important to observe that all the matrices have vanishing diagonal, moreover the matrices are pairwise orthogonal, therefore they are linearly independent.

If we find another Pauli triplet $\left(S_{1}, S_{2}, S_{3}\right)$ such that the diagonals are linearly independent, then we have a fifth algebra $\mathcal{A}_{5}$ such that $\left\{\mathcal{A}_{k}: 1 \leq k \leq 5\right\}$ spans linearly $M_{4}(\mathbb{C})$. Indeed, if $A$ is any matrix, then we can find $T \in \mathcal{A}_{5}$ such that $A-T$ has 0 diagonal and this is in the linear hull of $\left\{\mathcal{A}_{j}: 1 \leq j \leq 4\right\}$. It follows that the reduced densities in $\left\{\mathcal{A}_{j}: 1 \leq j \leq 5\right\}$ determines $\rho_{A B}$ uniquely. 
Here is an example of the above described triplet:

$$
\frac{1}{2}\left[\begin{array}{rrrr}
1 & 1 & 1 & 1 \\
1 & 1 & -1 & -1 \\
1 & -1 & -1 & 1 \\
1 & -1 & 1 & -1
\end{array}\right], \quad \frac{1}{2}\left[\begin{array}{rrrr}
1 & \mathrm{i} & \mathrm{i} & -1 \\
-\mathrm{i} & -1 & -1 & \mathrm{i} \\
-\mathrm{i} & -1 & 1 & -\mathrm{i} \\
-1 & -\mathrm{i} & \mathrm{i} & -1
\end{array}\right], \quad \frac{1}{2}\left[\begin{array}{rrrr}
-1 & \mathrm{i} & 1 & \mathrm{i} \\
-\mathrm{i} & 1 & \mathrm{i} & 1 \\
1 & -\mathrm{i} & 1 & \mathrm{i} \\
-\mathrm{i} & 1 & -\mathrm{i} & -1
\end{array}\right]
$$

These matrices are not elementary tensors (but they are Hadamard matrices [1] up to a constant multiple and were found by means of an exhaustive search algorithm on a computer).

Proposition 2 If all the matrices of the Pauli triplet generating the subalgebras $\mathcal{A}_{j}$ are of the form $\pm \sigma_{k} \otimes \sigma_{l}(0 \leq k, l \leq 3)$, then we need at least 6 triplets to span $M_{4}(\mathbb{C})$.

Proof. Assume that a Pauli triplet $\left(T_{1}, T_{2}, T_{3}\right)$ in $M_{4}(\mathbb{C})$ is such that every element is of the form $\pm \sigma_{i} \otimes \sigma_{j}(0 \leq i, j \leq 3)$.

If $T_{1}= \pm \sigma_{i} \otimes \sigma_{j}$ and $T_{2}= \pm \sigma_{k} \otimes \sigma_{l}$, then

$$
\begin{aligned}
\pm \mathrm{i} T_{3}= & -\sum_{m, n}\left(\epsilon_{i k m} \epsilon_{j l n} \sigma_{m} \otimes \sigma_{n}\right) \\
& +\mathrm{i}\left(\delta_{i k} \sum_{n}\left(\epsilon_{j l n} \sigma_{0} \otimes \sigma_{n}\right)+\delta_{j l} \sum_{m}\left(\epsilon_{i k m} \sigma_{m} \otimes \sigma_{0}\right)\right)+\delta_{i k} \delta_{j l} \sigma_{0} \otimes \sigma_{0} .
\end{aligned}
$$

Since $T_{3}$ is self-adjoint but $\mathrm{i} \sigma_{i} \otimes \sigma_{j}$ is not, it follows that exactly one of the relations $i=k$ and $j=l$ must hold. At least one of the operators $T_{i}$ should be of the form $\sigma_{0} \otimes \sigma_{j}$ or $\sigma_{j} \otimes \sigma_{0}$.

We have three operators in the form $\sigma_{0} \otimes \sigma_{j}$ and three in the form $\sigma_{j} \otimes \sigma_{0}(1 \leq j \leq 3)$. If we have 5 Pauli triplets, then at least one should contain two of the above tensor products (up to a sign). One can see that $\sigma_{0} \otimes \sigma_{j}$ and $\sigma_{i} \otimes \sigma_{0}$ cannot be in a triplet, therefore a triplet contains two operators in the form $\sigma_{0} \otimes \sigma_{j}$ or two operators like $\sigma_{j} \otimes \sigma_{0}$. In both cases, the third operator has similar form. Hence one of the operators $\sigma_{0} \otimes \sigma_{j}$ and $\sigma_{j} \otimes \sigma_{0}$ appears in two triplets and in this case 5 triplet cannot span the whole space.

Six subalgebras described in the proposition can be given by the following Pauli triplets:

$$
\begin{aligned}
& \left\{\sigma_{1} \otimes \sigma_{1}, \sigma_{1} \otimes \sigma_{2}, \sigma_{0} \otimes \sigma_{3}\right\}, \\
& \left\{\sigma_{2} \otimes \sigma_{2}, \sigma_{2} \otimes \sigma_{3}, \sigma_{0} \otimes \sigma_{1}\right\}, \\
& \left\{\sigma_{3} \otimes \sigma_{3}, \sigma_{3} \otimes \sigma_{1}, \sigma_{0} \otimes \sigma_{2}\right\}, \\
& \left\{\sigma_{2} \otimes \sigma_{2}, \sigma_{3} \otimes \sigma_{2}, \sigma_{1} \otimes \sigma_{0}\right\}, \\
& \left\{\sigma_{3} \otimes \sigma_{3}, \sigma_{1} \otimes \sigma_{3}, \sigma_{2} \otimes \sigma_{0}\right\}, \\
& \left\{\sigma_{1} \otimes \sigma_{1}, \sigma_{2} \otimes \sigma_{1}, \sigma_{3} \otimes \sigma_{0}\right\} .
\end{aligned}
$$

Together with $I$ each triplet linearly spans a subalgebra $\mathcal{A}_{j}(1 \leq j \leq 6)$ and the 6 subalgebras linearly span the whole $M_{2}(\mathbb{C}) \otimes M_{2}(\mathbb{C})$. 


\section{Generalizations}

Mutually unbiased bases (or measurements) are interesting from many point of view [4, 2, 13] and the maximal number of such bases is not completely known 12. The above discussed setting of state determination is somewhat similar. In this setting we may look for essentially orthogonal non-commutativ subalgebras while unbiased elementary measurement are given essentially orthogonal maximal Abelian subalgebras, see Prop. 2.2 of 8 . The next statement is an analogue of Parthasarathy's proposition.

Proposition 3 Let $\mathcal{A}_{1}$ and $\mathcal{A}_{2}$ be subalgebras of $M_{n}(\mathbb{C})$ and assume that they are isomorphic to $M_{k}(\mathbb{C})$. Then the following conditions are equivalent:

(i) If $P \in \mathcal{A}_{1}$ and $Q \in \mathcal{A}_{2}$ are minimal projections, then $\operatorname{Tr} P Q=n / k^{2}$.

(ii) The subspaces $\mathcal{A}_{1} \ominus \mathbb{C} I$ and $\mathcal{A}_{2} \ominus \mathbb{C} I$ are orthogonal in $M_{n}(\mathbb{C})$

Proof. It follows from the conditions that $n=m k$ and $\operatorname{Tr} P=\operatorname{Tr} Q=m$ for the minimal projections. Therefore, condition (i) is equivalent to $\operatorname{Tr}(I-k P)(I-k Q)=0$ which means that $(I-k P) \perp(I-k Q)$. Since the subspaces in (ii) are linearly spanned by these operators, the proposition follows.

Now we generalize Prop. 2 for $n$ qubits.

Proposition 4 If all the matrices of the Pauli triplet generating the subalgebras $\mathcal{A}_{j}$ of $M_{2^{n}}(\mathbb{C})$ are of the form $\pm \sigma_{k(1)} \otimes \ldots \otimes \sigma_{k(n)}(0 \leq k(i) \leq 3,1 \leq i \leq n)$, then we need more than $\left(2^{2 n}-1\right) / 3$ triplets to span $M_{2^{n}}(\mathbb{C})$.

Proof. First note that a matrix $\pm \sigma_{k(1)} \otimes \ldots \otimes \sigma_{k(n)}$ has only real elements or only imaginary elements. Among the three matrices of a Pauli triplet $\left(T_{1}, T_{2}, T_{3}\right)$, there is one imaginary or there are three imaginary matrices. Let $N$ be the number of triplets with 1 imaginary matrix and $M$ be the number of triplets with 3 imaginary ones. If the $N+M$ triplets with identity linearly span the self-adjoint subspace, then $3(N+M)+1 \geq 2^{2 n}$. Assume that

$$
N+M=\frac{2^{2 n}-1}{3}
$$

Since the dimension of the subspace of self-adjoint matrices with imaginary elements is $\left(2^{2 n}-2^{n}\right) / 2$, we must have

$$
N+3 M=\frac{2^{2 n}-2^{n}}{2} \text {. }
$$

One can see that equations (44) and (5) do not have integer solution.

We call a family $\mathcal{M}_{1}, \mathcal{M}_{2}, \ldots, \mathcal{M}_{d}$ of subalgebras strongly mutually unbiased if the conditions in the proposition hold for any pair. The maximal number of strongly mutually unbiased subalgebras is not know to us even in the simplest case when the large algebra is $M_{4}(\mathbb{C})$ and the subalgebras are isomorphic to $M_{2}(\mathbb{C})$. 
Following [8], we may call a family $\mathcal{M}_{1}, \mathcal{M}_{2}, \ldots, \mathcal{M}_{d}$ of subalgebras weakly mutually unbiased if the subspaces $\mathcal{M}_{1} \ominus \mathbb{C} I, \mathcal{M}_{2} \ominus \mathbb{C} I, \ldots, \mathcal{M}_{d} \ominus \mathbb{C} I$ are linearly independent. We showed that when the large algebra is $M_{4}(\mathbb{C})$ and the subalgebras are isomorphic to $M_{2}(\mathbb{C})$, then the maximum number of weakly unbiased subalgebras is 5 .

\section{Discussion and conclusions}

The optimal state tomography has been studied for a composite system of two qubits when measurements can be performed on one of the qubits and interactions of the two qubits can be implemented. Equivalently, we found physically realizable minimal set of reduced densities. The transformation described by (11) is realized by a properly designed measurement apparatus (see the experimental devices in [9]). Therefore, an additional, but still unsolved problem is to find the unitary to each of the reduced densities that transforms the state to be measured to another one belonging to the reduced density in question. (Some preliminary results on the effect of the real 4-dimensional rotation matrices on the reduced densities is found in [10].)

The construction of 5 Pauli triplets of $4 \times 4$ matrices from the tensor products of Pauli matrices contains heuristic steps combined with an exhaustive search for the missing final triplet. This makes it practically impossible to generalize the method for higher dimensions. Even if the heuristic steps were removed the resulted algorithm would fall to the NP-hard category because of the exhaustive search.

The questions we posed for two qubits can be asked about three (or more qubits). The dimension of $M_{8}(\mathbb{C}) \ominus \mathbb{C} I$ is 63 , so we need at least 21 Pauli triplets to span the whole space. In the moment we can construct 22 spanning Pauli triplets by ad hoc method (this will be discussed in another publication).

The problem investigated here motivates the definition of strongly and weakly unbiased subalgebras. In relation with them, several questions can be raised.

\section{References}

[1] J.A. Bergou, V. Buzek, E. Feldman, U. Herzog and M. Hillery, Programmable quantum state discriminators with simple programs, arXiv:quant-ph/0602164, 2006.

[2] P.O Boykin, M. Sitharam, P.H. Tiep and P. Wocjan, Mutually unbiased bases and orthogonal decompositions of Lie algebras, arXiv:quant-ph/0506089, 2005.

[3] A. Isidoni, Nonlinear Control Systems, Springer-Verlag, Berlin, 1995.

[4] K. Kraus, Complementarity and uncertainty relations, Phys. Rev. D. 35, 3070-3075, 1987. 
[5] M. A. Nielsen and I. L. Chuang, Quantum Computation and Quantum Information, Cambridge University Press, 2000.

[6] M. Ohya and D. Petz, Quantum Entropy and Its Use, Springer-Verlag, Berlin, 1993.

[7] M.G.A. Paris and J. Rehácek (eds), Quantum State Estimation, Lecture Notes in Physics, Springer-Verlag, 2004.

[8] K.R. Parthasarathy, On estimating the state of a finite level quantum system, Infinite Dimensional Analysis, Quantum Probability and Related Topics, 7, 607-617. 2004.

[9] J. Rehácek, B. Englert and D. Kaszlikowski, Minimal qubit tomography, Physical Review A 70, 052321, 2004.

[10] A. Szántó, The reconstruction of the state of two qubits (in Hungarian), Student Research Report, BUTE, 2005.

[11] W. Tadej and K. Zyczkowski, A concise guide to complex Hadamard matrices, arXiv:quant-ph/0512154, 2005.

[12] R. Werner, http://www.imaph.tu-bs.de/qi/problems/13.html

[13] W.K. Wooters and B.D. Fields, Optimal state determination by mutually unbiased measurements, Annals of Physics, 191, 363-381, 1989. 